\title{
Radio signatures of CME-streamer interaction and source diagnostics of type II radio burst
}

\author{
S. W. Feng ${ }^{1,2,3}$, Y. Chen ${ }^{1}$, X. L. Kong ${ }^{1}$, G. Li ${ }^{4,1}$, H. Q. Song ${ }^{1}$, X. S. Feng ${ }^{2}$, and Ying Liu ${ }^{5}$
}

\begin{abstract}
It has been suggested that type II radio bursts are due to energetic electrons accelerated at coronal shocks. Radio observations, however, have poor or no spatial resolutions to pinpoint the exact acceleration locations of these electrons. In this paper, we discuss a promising approach to infer the electron acceleration location by combining radio and white light observations. The key assumption is to relate specific morphological features (e.g. spectral bumps) of the dynamic spectra of type II radio bursts, to imaging features (e.g. CME going into a streamer) along the CME (and its driven shock) propagation. In this study, we examine the CME-streamer interaction for the solar eruption dated on 2003 November 1 . The presence of spectral bump in the relevant type II radio burst is identified, which is interpreted as a natural result of the shock-radio emitting region entering the dense streamer structure. The study is useful for further determinations of the location of type II radio burst and the associated electron acceleration by CME-driven shock.
\end{abstract}

Subject headings: shock waves - Sun: coronal mass ejections (CME) - Sun: radio radiation - Sun: corona

\section{Introduction}

Type II solar radio bursts are narrow stripes present in the metric to kilo-metric wavelength range drifting gradually from higher to lower frequencies as revealed from the solar

\footnotetext{
${ }^{1}$ Shandong Provincial Key Laboratory of Optical Astronomy and Solar-Terrestrial Environment, School of Space Science and Physics, Shandong University at Weihai, Weihai 264209, China

${ }^{2}$ SIGMA Weather Group, State Key laboratory for Space Weather, Center for Space Science and Applied Research, Chinese Academy of Sciences, Beijing 100190, China

${ }^{3}$ College of Earth Sciences, Graduate School of Chinese Academy of Sciences, Beijing 100049, China

${ }^{4}$ Department of Physics and CSPAR, University of Alabama in Huntsville

${ }^{5}$ Space Sciences Laboratory, University of California, Berkeley, CA 94720, USA
} 
radio dynamic spectra (Wild 1950; Wild et al., 1954; Nelson \& Melrose, 1985). It is generally believed that these bursts are excited by energetic electrons accelerated at magnetohydrodynamic (MHD) shocks driven by solar eruptions via the plasma emission process (Ginzburg \& Zheleznyakov, 1958). The emitting frequencies are close to the local plasma frequency and/or its harmonic. There exists a large body of works in the literature studying this type of solar radio bursts (see, e.g., Dulk, 1985; Pick \& Vilmer, 2008, and references therein). Nevertheless, the generation mechanism and the source location of coronal metric type II radio bursts remain as a fiercely debated problem. The focus of the debate is whether coronal metric type II bursts are associated with blast waves driven by the flare heating process, or with a piston-driven shock at the CME nose front and/or flank (e.g., Cliver et al., 1999; Oh et al., 2007; Vršnak \& Cliver, 2008; Magdalenić et al., 2008; Liu et al., 2009). At present, the most direct method to resolve this issue is via radio imaging observations with radioheliographs, like the Teepee Tee Array of the Clark Lake Radio Observatory, the Culgoora Radioheliograph, and the Nancay Radioheliograph (e.g., Gergely et al., 1983; Maia et al., 2000; for reviews see Pick, 1999; Pick \& Vilmer, 2008). However, simultaneous observations of white light/EUV (extreme ultraviolet) imagings of CME shocks and metric type II radio imagings have been rare.

A promising approach to resolve the above issue is to establish physical connections between the spectral shape from the radio data and certain eruptive processes observed using solar imaging instruments in the white light and EUV wavelengths. Since the type II emission frequency is largely determined by the coronal electron density along the shock path, any coronal density variation along the path will affect the shape of the radio emission in the corresponding dynamic spectrum. It is well known that coronal streamers are the brightest dense structure extending all the way from the solar surface to interplanetary space, frequently interacting with the CME ejecta and related disturbances (e.g., Subramanina et al.,1999; Sheeley et al., 2000; Wang \& Sheeley, 2007; Bemporad et al., 2008, 2010; Chen et al., 2010; Feng et al., 2011). Previous studies on radio bursts (e.g., Reiner et al., 2003; Cho et al., 2007, 2008, 2011) have illustrated the importance of CME-streamer interaction region as a source for type II bursts.

In a recent study, Kong et al. (2012) reported an interesting type II event occurred on 2011 March 27. In that event the type II radio emission showed a "sudden" transition from a relatively slow drift to a much fast drift. By analyzing simultaneous EUV and coronagraph imaging observations, Kong et al. (2012) inferred that the observed sudden spectral transition was a result of the transit of an eruption-driven shock across the streamer boundary, where the density drops sharply, from inside of the streamer. In this study we examine another type II event which shows a "bump-like" feature instead of a "break". We argue that such a feature is a result of a CME-driven shock 
crossing the dense streamer from outside.

According to previous observational and numerical studies, the streamer can be several times denser than surrounding solar wind environment (e.g., Habbal et al., 1997; Parenti et al., 2000; Strachan et al., 2002; Chen et al., 2001; Li et al., 2006). Therefore, the passage of a shock crossing a nearby streamer can result in a noticeable effect on the shape of the type II emission in the dynamic spectrum. Generally speaking, the type II strips may deviate away from their preceding declining trend and get elevated temporarily depending on the shock speed, the transit direction, and the exact density gradient along the shock path.

It is the aim of this study to identify a type II radio burst that bears the afore-mentioned evolutionary feature in the dynamic spectrum, and to establish the physical connection of the feature to eruptive processes recorded by coronagraphs, and to further infer the electron accelerating site responsible for the radio burst.

The paper is organized as follows. In Section 2, we briefly discuss the solar radio spectrographs and coronagraphs used in the study. In Section 3 we present the type II event, determine its relationship with the associated mass eruption, estimate the radio source, and finally consider other radio features possibly relevant to the CME-driven shock-streamer interaction. A summary with some brief discussion is provided in Section 4.

\section{Observational data}

The radio data used in this study are from WIND/WAVES (Bougeret et al., 1995), the Bruny Island Radio Spectrograph (BIRS; Erickson, 1997), and the Learmonth (LEAR;

Kennewell \& Steward, 2003). The ranges of frequency coverage and temporal resolution are listed in the second to fourth columns of Table 1. The corona imaging data are from LASCO (Large Angle Spectrographic Coronagraph) C2 on board the SOHO (Solar and Heliospheric Observatory) Spacecraft (Brueckner et al., 1995) and the Mark-IV (MK4) coronagraph operated by Mauna Loa Solar Observatory (Elmore et al., 2003). Their fields of view and observational cadences are given in the last two columns of Table 1. Previous radio imaging studies have shown that the metric type II bursts can start as low as a few tenths of solar radii above the photosphere (e.g. Pohjolainen et al. 2008; Magdalenić et al. 2008; Nindos et al. 2011), so we focus mainly on the MK4 observation in our study. 


\section{Radio burst and CME-streamer interaction on 2003 November 1}

On 2003 November 1, an M3.2 solar flare from NOAA AR10486 erupted from S12W60 in heliographic coordinates. The flare eruption was between 22:26 UT - 22:49 UT with the GOES X-ray flux peaked at 22:36 UT. The associated CME was first observed by MK4 and LASCO C2 at 22:33:06 UT and 23:06:53 UT as shown in Figures 1(a) and 1(b). The arrows indicate the location of the CME leading edges which were $1.3 \mathrm{R}_{\odot}$ and $3.8 \mathrm{R}_{\odot}$. Their CPAs (central position angles) were $260^{\circ}$ and $245^{\circ}$, respectively. From these data we can deduce that the CME nose propagated slightly non-radially with a linear speed of 878 $\mathrm{km} \mathrm{s}^{-1}$. According to the online CDAW (Coordinated Data Analysis Workshops) catalog (Yashiro et al., 2004), the CME linear speed was $899 \mathrm{~km} \mathrm{~s}^{-1}$, consistent with the above estimate. Figure 1(c) presents the MK4 data at 22:45:50 UT. We can see that the CME erupted from within a multi-streamer system with a bright arc-like leading edge, which expanded rapidly from 22:33:06 UT to 22:44:50 UT. During the expansion, the CME pushed the surrounding streamers aside. Figure $1(\mathrm{~d})$ is the difference image obtained from the LASCO C2 observations at 23:06:53 UT and 22:30:05 UT. According to this image and the one obtained later a diffusive structure in front of the bright CME ejecta can be clearly identified. We believe that this structure corresponds to the shock driven by the eruption (c.f., Vourlidas et al., 2003). There were two preceding CMEs, first observed in C2 field of view at 14:54:05 UT and 21:30:08 UT with CPAs being about $274^{\circ}$ and $318^{\circ}$, respectively. We discuss the connection between these two events and our event at the start of Sec. 3.2.

\subsection{Spectral bump of the type II radio burst}

In Figure 2, we show the radio dynamic spectrum observed during the above-mentioned solar eruption. The spectrum is given by combining the data from all three radio spectrographs listed in Table 1. There was an obvious type III burst at 22:33 UT, followed by two strong stripes of type II emission corresponding to the fundamental (F) and harmonic (H) branches. The type II lasted for about 28 minutes extending from $\sim 140 \mathrm{MHz}$ to 10 MHz. The average frequency drift rate of the $\mathrm{F}$ branch was $\sim-0.08 \mathrm{MHz} \mathrm{s}^{-1}$. In the paper of Cane \& Erickson (2005), this burst was taken as a typical metric type II event with a relatively large and continuous frequency coverage. In this study, we focus on the radio features observed between 22:44 UT and 22:54 UT.

At 22:44 UT, both F and $\mathrm{H}$ branches started to be intermittent and this intermittence lasted till 22:48 UT, when the emission became continuous again with a clear band splitting for the H branch. Band splitting phenomenon has been discussed, e.g., by Smerd (1974) and 
Vrsňak et al. (2001). In the mean time, a large change of the spectral slope was clearly seen at about $\sim 40 \mathrm{MHz}$ of the $\mathrm{H}$ branch. The average frequency drift of this branch, $\sim-0.4 \mathrm{MHz} \mathrm{s}^{-1}$ between 22:34 UT and 22:44 UT, decreased to $\sim-0.04 \mathrm{MHz} \mathrm{s}^{-1}$ during the spectral plateau from 22:44 UT to 22:52 UT. After the plateau, the magnitude of the average drift rate increased slightly to $\sim 0.06 \mathrm{MHz} \mathrm{s}^{-1}$. The two solid-dashed lines in the figure are fittings to the temporal evolution of the $\mathrm{F}$ and $\mathrm{H}$ branches before 22:44 UT using the one-fold Saito density model (Saito, 1970) with a radial shock propagation speed of 900

$\mathrm{km} \mathrm{s}^{-1}$. The spectral plateau lies clearly above the black curves, which we define as the type II spectral bump. It is the main focus of this study.

\subsection{CME-streamer interaction and the type II spectral bump}

Since the emission frequency of type II bursts depends on the plasma density along the shock path, the most straightforward explanation of the spectral bump is that the shock was passing through a high density coronal structure. It is possible that such dense structure could be coronal disturbances caused by preceding eruptions. Indeed, as mentioned before, there existed two preceding CMEs to our CME. The first CME took place $\sim 8$ hours before our CME. That CME is possibly too early to affect the shape of the studied type II radio emission. This is confirmed by the online MK4 animations for both the white-light and difference data in the time period of 17:22:29 UT to 22:00:37 UT. We can see that there were no observable signatures of this CME in the MK4 FOV from the beginning of the animations. The second event did not cause strong disturbances to the equatorial streamer as a result of its source location being outside of the streamer and its limited angular span. The CME left the MK4 FOV 30 minutes before the start of the radio bursts. Therefore, it is unlikely that the high density structure accounting for the observed spectral bump was directly associated with the disturbances of these two earlier eruptions.

According to the coronagraph observation, the CME collided with nearby streamers from both flanks. Therefore, interaction of shock wave with both the northern and southern streamers can cause the spectral bump. To further discern the interaction with which streamer is responsible for the type II spectral bump, in Figure 3 we present ten base-difference images (coronal images subtracted the pre-eruption corona image at 22:30:06 UT) from MK4 observation for the period of 22:33:06 UT to 23:59:35 UT with the cadence of 3 minutes.

We first analyze the details of the CME (shock) interaction with both streamers. As seen from Figure 3 and the online animations, both the spatial location of streamers and the CME-propagation seem to be asymmetric. The shock wave first 
encountered northern streamer and a few minutes later also southern streamer. Black arrows in the figure indicate the locations where the streamer deflections were first observed. The deflections were present even in the last panel of this figure. As measured from Figure 3(c), the stand-off distance of the shock along the northern streamer is about $0.3 \mathrm{R}_{\odot}$. From Figure $3(\mathrm{~d})$, the southern streamer seemed to be first deflected by the CME shock wave at 22:41:54 UT. The deflection was very weak yet noticeable (see online animation) and it can be considered as the first signature of the interaction between the shock and the southern streamer. Note that the spectral bump started around 22:46 UT. The lack of the change in the radio emission during the interaction of the shock and the northern streamer suggests that the type II radio emission came from the southern part of the shock wave. This is further supported by the following analyses.

We also compare the shock heights measured from the MK4 observation and that deduced from the type II emission using the Saito density model in Figure 4(a). The shock heights are shown as bars whose length is due to uncertainties of the density associated with the type II band width. To measure the heliocentric distances of the northern and southern parts of the CME shock wave, we draw two lines starting from the source of the eruption passing through the disturbed northern and southern streamers, respectively, and determine the heliocentric distances of the intersection points of both lines with the shock wave. The two lines are plotted as solid and dashed lines in the upper panels of Figure 3, and the obtained distances of the shock wave are shown in Figure 4(a) as pluses and asterisks. We note that there are uncertainties in measuring these distances due to the quality of the MK4 data. However, the large difference between the distances along the two directions is significant. Therefore, we conclude that the shock distances along the dashed line agree with the radio shock heights. In other words, the radio source is likely located at the southern part of the shock, rather than the northern part. This strongly favors the interaction of the shock wave with the southern streamer being the cause of the spectral bump.

To confirm the applicability of the Saito model to this event, in Figure 4(b) we plot the radial electron density profiles deduced from the MK4 polarized brightness (pB) data measured before the eruption at 22:30:06 UT. The profiles are along 3 position angles (PAs) at 230, 240, and 270 degrees. We use the standard pB inversion package from the SolarSoftWare (SSW). The Saito density model is also shown as the solid line. Below $1.6 \mathrm{R}_{\odot}$, all three sets of the $\mathrm{pB}$ density profiles are considerably close to the Saito model. This indicates the applicability of this model in a range of PAs, therefore the above conclusion should not be affected by the significant non-radial propagation of the shock wave.

We now compare the estimated time of the shock transit across the streamer with the 
bump duration. If the radio bumps were caused by the shock passing through the southern streamer, it had to be the shock flank, not the nose. Assuming a propagation distance of the shock inside the streamer being $0.5 \mathrm{R}_{\odot}-1 \mathrm{R}_{\odot}$ (reasonable values for width of a streamer), and a shock speed of $800 \mathrm{~km} \mathrm{~s}^{-1}$, then the transit will take 7.2 to 14.5 minutes. This is also consistent with the 10-minute duration of the spectral bump.

In summary, we believe that the bumped type II burst in this event is caused by electrons accelerated at the southern flank of the CME-driven shock, and the bump is due to the shock propagating through the dense streamer. Figure 5 is a cartoon illustrating our understanding of this event. The outward propagating CME-driven shock is denoted by the red solid-dashed curve and the radio source by thick segment. We can see that the streamer transit of the radio source took place between 22:45 UT to 22:55 UT. This transit accounted for the spectral bump reported above. We can also see that the radio-emitting part of the shock is likely quasi-perpendicular. We will return to this point in the discussion section.

Last, we have pointed out that the first signature of the interaction of the shock flank with the southern streamer seemed to occur $\sim 4$ minutes before the start of the spectral bump. This can be understood if the radio source region (and therefore the associated energetic electron acceleration region) is not at the edge of the shock flank, which entered the streamer first.

\subsection{Other radio features observed during the spectral bump}

In this subsection, we report other radio features observed in the dynamic spectrum of this event. Since the radio data available provide no spatial information of radio signals, these features do not necessarily have causal relationship with the spectral bump. We include them here because they occurred temporally close to the spectral bump.

From the dynamic spectrum shown in Figure 2, we observe diffusive type IV radio emission with frequencies above $70 \mathrm{MHz}$ starting around 22:42 UT, at which time the $\mathrm{H}$ branch of the type II burst became intermittent and split while the F branch almost disappeared. It seems that this type IV burst lasted significantly longer than the duration of the type II bump. In addition, two episodes of type III-like bursts were present at 22:48 UT and 22:49 UT with frequencies starting at $>180 \mathrm{MHz}$. It is not possible to determine the drifting direction of these type III-like bursts. If they had positive drifts, i.e., they were reversal (RS) type III-like, it would imply the existence of precipitating electrons (from shock region). On the other hand, there was a strong type III burst, starting at about 22:47 UT, right below the bump in the dynamic spectrum. This lower type III burst is possibly due to 
shock-accelerated electrons that are released into open field lines during the shock-streamer interaction process. Motions of energetic electrons accounting for the above-mentioned type IIIs and type IV emissions have been plotted in the cartoon shown in Figure 5.

\section{Conclusions and discussion}

In this paper we explore the possibility of using particular features (spectral bump in this study) of type II radio bursts to infer the underlying electron acceleration sites. In the event, we have identified clear frequency bump in the type II dynamic spectrum, which was interpreted as a result of the CME shock entering nearby dense streamer structure. We propose that the type II radio burst included here was closely associated with CME-driven shock-streamer interaction. We also documented a few possibly-relevant radio signatures including emissions of type IV bursts above and type III-like bursts above and below the type II spectral bump.

As the radio emitting region carried by the shock approaches the dense streamer structure, both the $\mathrm{F}$ and $\mathrm{H}$ bands can get absorbed or reflected depending on the exact density gradient along the observing line of sight. This may explain the intermittency or disappearance of radio signals before or at the early stage of the type II bump. The streamer region is denser and slower than the surrounding solar wind plasmas. A shock propagated into a streamer can therefore be strengthened. This will affect the electron acceleration process and the consequent radio emission intensity.

The observed type IV burst may be excited by electrons that are accelerated and released by the shock into the confining streamer structure during the interaction. The type III bursts observed above and below the bump may also be produced by shock-accelerated electrons, which escaped from the shock propagating anti-sunwards or sunwards along open or largescale closed field lines during the shock-streamer interaction. Further studies on the effect of the shock-streamer interaction on electron dynamics and radio emission are required for a better understanding of the radio features presented here.

One implication of this study is that the CME-driven shock flank are important sources of type II bursts. This is consistent with previous studies (e.g., Reiner et al., 2003; Cho et al., 2011). At the flank, the initial lateral expansion of the ejecta is very fast and can drive a shock there. Since field lines near the surface of the Sun are largely radial, the shock geometry at the shock flank is likely quasi-perpendicular. Electron acceleration at a quasi-perpendicular shock has been the subject of previous theoretical and simulation works (Wu, 1984; Lee et al., 1996; Zank et al., 1996; Guo \& Giacalone, 2010), as well as some 
observational analyses (Holman \& Pesses, 1983; Bale et al., 1999). It was suggested that a quasi-perpendicular shock is an effective electron accelerator. However, it should be noted that coronagraph images, upon which the study is based, are two-dimensional projection of the complex three-dimensional eruptive processes, structures that are not relevant to our study may appear as relevant. And it is generally not possible to determine the exact coronal shock geometry with available data sets.

The study presented here provides an indirect way of inferring the acceleration site of electrons along the shock surface. This can be important to understanding the effect of shock geometry on the electron acceleration process. In the future, we plan to extend our studies to examine more events with spectral bumps and understand their physical connection to the CME (shock)-streamer interactions.

We are grateful to the STEREO, SOHO/LASCO, MLSO/MK4, Wind/Waves, and LEAR teams for making their data available online. We thank Dr. Stephen White and Dr. Bill Erickson for providing the BIRS data. This work was supported by 973 program 2012CB825601, NNSFC grants 40825014, 40890162, 41028004, and the Specialized Research Fund for State Key Laboratory of Space Weather in China. H. Q. Song was also supported by NNSFC 41104113. GL's work at UAHuntsivlle was supported by NSF CAREER: ATM0847719 and NSF SHINE: AGS-0962658. GL would also like to thank YC and his group for their hospitality during his visit at SDU at Weihai.

\section{REFERENCES}

Bale, S. D., Reiner, M. J., Bougeret, J. L., Kaiser, M. L., Krucker, S., Larson, D. E., Lin, R. P. 1999, GRL, 26, 1573

Bemporad, A., Poletto, G., Landini, F., \& Romoli, M. 2008, Ann. Geophys., 26, 3017

Bemporad, A., Soenen, A., Jacobs, C., Landini, F., \& Poedts, S. 2010, ApJ, 718, 251

Bougeret, J. L., Kaiser, M. L., Kellogg, P. J., Manning, R., Goetz, K., Monson, S. J., Monge, N., Friel, L., Meetre, C. A., \& Perche, C. 1995, Space Sci. Rev., 71, 231

Brueckner, G. E., Howard, R. A., Koomen, M. J., Korendyke, C. M., Michels, D. J., Moses, J. D., Socker, D. G., Dere, K. P., Lamy, P. L., \& Llebaria, A. et al. 1995, Solar Phys., $162,357$.

Cane, H. V., \& Erickson, W. C. 2005, ApJ, 623, 1180 
Chen, Y., \& Hu, Y. Q. 2001, Sol. Phys., 199, 371

Chen, Y., Song, H. Q., Li, B., Xia, L. D., Wu, Z., Fu, H., \& Li, X. 2010, ApJ, 714, 644

Cho, K. S., Lee, J., Moon, Y. J., Dryer, M., Bong, S. C., Kim, Y. H., \& Park, Y. D. 2007, A\&A, 461, 1121

Cho, K. S., Bong, S. C., Kim, Y. H., Moon, Y. J., Dryer, M., Shanmugaraju, A., Lee, J., \& Park, Y. D. 2008, A\&A, 491, 873

Cho, K. S., Bong, S. C., Moon, Y. J., Shanmugaraju, A., Kwon, R. Y., \& Park, Y. D. 2011, A\&A, 530, 16

Cliver, E. W., Webb, D. F., \& Howard, R. A. 1999, Sol. Phys, 187,89

Dulk, G. A. 1985, ARA\&A, 23, 169

Elmore, D. F., Burkepile, J. T., Darnell, J. A., Lecinski, A. R., \& Stanger, A. L. 2003, Proc. SPIE, 4843

Erickson, W. C. 1997, Publication Astronomical Society of Austraila, 14, 3, 278-282

Feng, S. W., Chen, Y., Li, B., Song, H. Q., Kong, X. L., Xia, L. D., \& Feng, X. S. 2011, Sol. Phys., 272, 119

Gergely, T. E., Kundu, M. R., Hildner, E. 1983, Sol. Phys., 268, 403

Ginzburg, V. L., \& Zhelezniakov, V. V. 1958, SvA, 2, 653

Guo, F., \& Giacalone, J. 2010, ApJ, 715, 406

Habbal, S. R., Woo, R., Fineschi, S., O’Neal, R., Kohl, J., Noci, G., \& Korendyke, C. 1997, ApJ, 489, 103

Holman, G. D., \& Pesses, M. E. 1983, ApJ, 267, 837

Kennewell, J. \& Steward, G. 2003, Solar Radio Spectrograph [SRS] Data Viewer [Srsdisplay] (Sydney: IPS Radio and Space Serv.), ftp://ftp.ips.gov.au/wdc-data/spec/doc/Other\%20Document/Srsdispl.doc

Kong, X. L., Chen, Y., Feng, S. W., Song, H. Q., Li, G., Guo, F., Jiao, F. R. 2012, in press Lee, M., Shapiro, V. D., \& Sagdeev, R. Z. 1996, J. Geophys. Res., 101, 4777

Li, B., \& Li, X. 2006, A\&A, 456, 359 
Liu, Y., Luhmann, J. G., Bale, S. D., \& Lin, R.P. 2009, ApJ, 691, L151

Magdalenić, J., Vršnak, B., Pohjolainen, S., Temmer, M., Aurass, H., Lehtinen, N. J. 2008, Sol. Phys., 253, 305

Maia, D., Pick, M., Vourlidas, A., Howard, R. 2000, ApJ, 528, 49

Nelson, G. J., \& Melrose, D. B. 1985, Solar Radiophysics: Studies of Emission from the Sun at Metre Wavelengths, ed. D. J. McLean \& N. R. Labrum (Cambridge: Cambridge Univ. Press), 333

Nindos, A., Alissandrakis, C.E., Hillaris, A., Perka-Papadema, P. 2011, A\&A, 531, 31

Oh, S. Y., Yi, Y., Kim, Y. H. 2007, Sol. Phys., 245, 391

Parenti, S., Bromage, B. J. I., Poletto, G., Noci, G., Raymond, J. C., \& Bromage, G. E. 2000, A\&A, 363, 800

Pick, M., 1999, in Proc. Nobeyama Symp., ed. T. Bastion, N. Gopalswamy, \& K. Shibasaki (NRO Rep. 479; Kiysato: Nobeyama Radio Obs.), 187

Pick, M., \& Vilmer, N. 2008, A\&ARv, 16, 1

Pohjolainen, S. 2008, A\&A, 483, 297

Reiner, M. J., Vourlidas, A., \& Cyr, O. C. St. et al. 2003, ApJ, 590, 533

Saito, K., 1970, Ann. Tokyo Astr. Obs., 12, 53

Sheeley, N. R., Hakala, W. N., Wang, Y. M. 2000, J. Geophys. Res. 105, 5081

Smerd, S. F., Sheridan, K. V., \& Stewart, R. T. 1974, in IAU Symp. 57, ed. G. A. Newkirk, 389

Strachan, L., Suleiman, R., Panasyuk, A. V., Biesecker, D. A., \& Kohl, J. L. 2002, ApJ, 571, 1008

Subramanian, P., Dere, K. P., Rich, N. B., \& Howard, R. A. 1999, J. Geophys. Res., 104, 321

Vourlidas, A., Wu, S. T., Wang, A. H., Subramanian, P., \& Howard, R. A. 2003, ApJ, 598, 1392

Vršnak, B., Aurass, H., Magdalenic, J., Gopalswamy, N. 2001, A\&A, 377, 321 
Vršnak, B., Cliver, E. W. 2008, Sol. Phys., 253, 215

Wang, Y. M., Sheeley, N. R. Jr., \& Rich, N. B. 2007, ApJ, 658, 1340

Wild, J. P., \& McCready, L. L. 1950, Aust. J. Phys. 3, 387

Wild, J. P., Murray, J. D., Rowe, W. C. 1954, Aust. J. Phys. 7, 439

Wu, C. S. 1984, J. Geophys. Res., 89(A10), 8857

Yashiro, S., Gopalswamy, N., Michalek, G., St. Cyr, O. C., Plunkett, S. P., Rich, N. B., Howard, R. A., J. Geophys. Res., 2004, 109(A7), 07105

Zank, G. P., Pauls, H. L., Cairns, I. H., \& Webb, G. M. 1996, J. Geophys. Res., 101, 457 
Table 1: Parameters of the instruments used in this study. Ranges of frequency coverage and temporal resolution of the radio spectrographs are listed in the second to fourth columns, fields of view and temporal cadences of the coronagraphs are listed in the last two columns.

\begin{tabular}{lcccccc}
\hline \hline \multirow{2}{*}{ Instruments } & \multicolumn{3}{c}{ Radio spectrograph } & & \multirow{2}{*}{ Coronagraph } & \\
\cline { 2 - 3 } \cline { 6 - 7 } & WAVES & BIRS & LEAR & & MK4 & LASCO \\
& RAD2 & & & & C2 \\
\hline Observational & $1.07-13.8$ & $5-62.5$ & $25-180$ & & $1.14-2.85$ & $2.1-6.0$ \\
range & $(\mathrm{MHz})$ & $(\mathrm{MHz})$ & $(\mathrm{MHz})$ & & $\left(\mathrm{R}_{\odot}\right)$ & $\left(\mathrm{R}_{\odot}\right)$ \\
\hline Temporal & 60 & 3 & 3 & & 3 & $12-36$ \\
resolution & $(\mathrm{sec})$ & $(\mathrm{sec})$ & $(\mathrm{sec})$ & & $(\mathrm{min})$ & $(\mathrm{min})$ \\
\hline
\end{tabular}

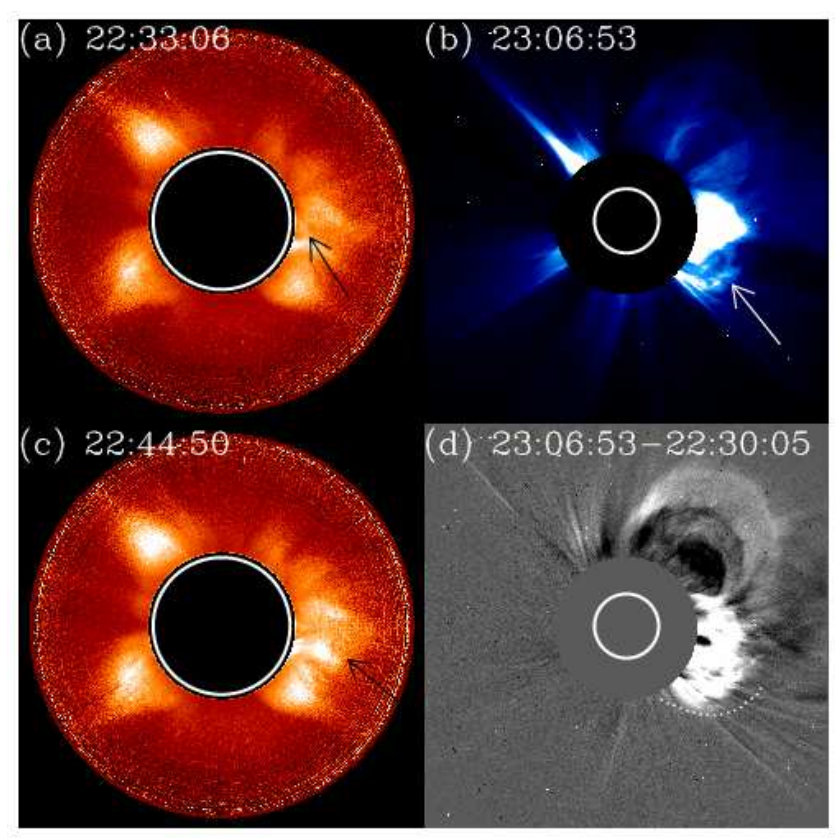

Fig. 1. - Coronal images for the 2003 November 1 event observed by MK4 and LASCO C2 coronagraphs $(\mathrm{a}-\mathrm{c})$. The arrows denote the location of the CME fronts. Panel $(\mathrm{d})$ is the difference image obtained from the LASCO C2 observations at 23:06:53 UT and 22:30:05 UT. Dotted curve in (d) plots the diffusive shock ahead of the bright CME ejecta on the day. The CME erupts from within a multi-streamer system with a bright arc-like front, pushing aside the surrounding streamers during its rapid expansion and propagation. (Animations of this figure are available in the online journal.) 


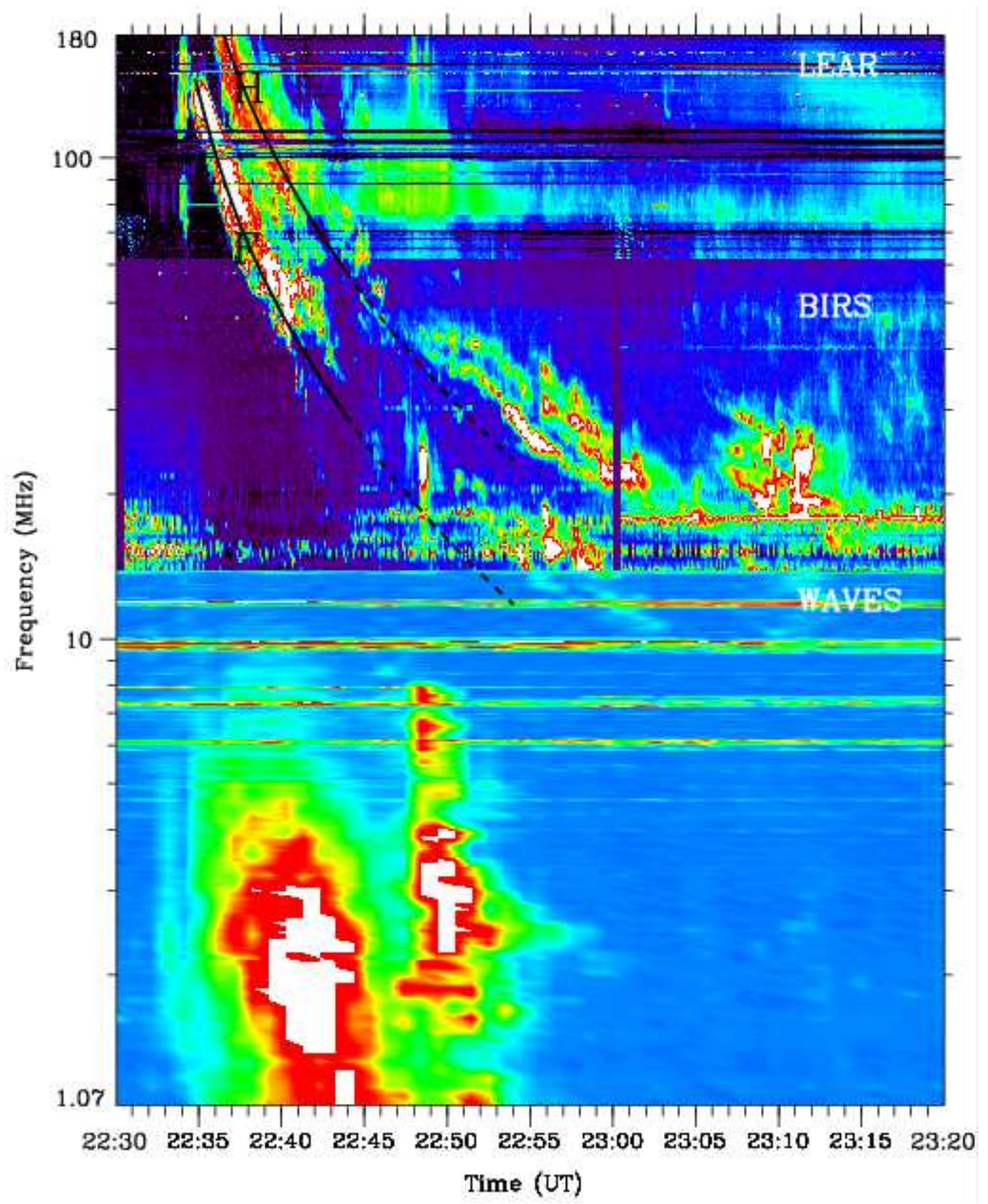

Fig. 2. - The radio dynamic spectrum on 2003 November 1 from 22:30 UT to 23:20 UT, given by the composition of the spectra obtained by WAVES (1.07-13.8 MHz), BIRS (13.8 - $62.5 \mathrm{MHz})$, and LEAR (62.5 - $180 \mathrm{MHz})$. F and $\mathrm{H}$ denote the fundamental and harmonic branches of the burst. The two solid-dashed lines are fittings to the radio spectrum before 22:44 UT using the one-fold Saito model and a shock speed of $900 \mathrm{~km} \mathrm{~s}^{-1}$. 


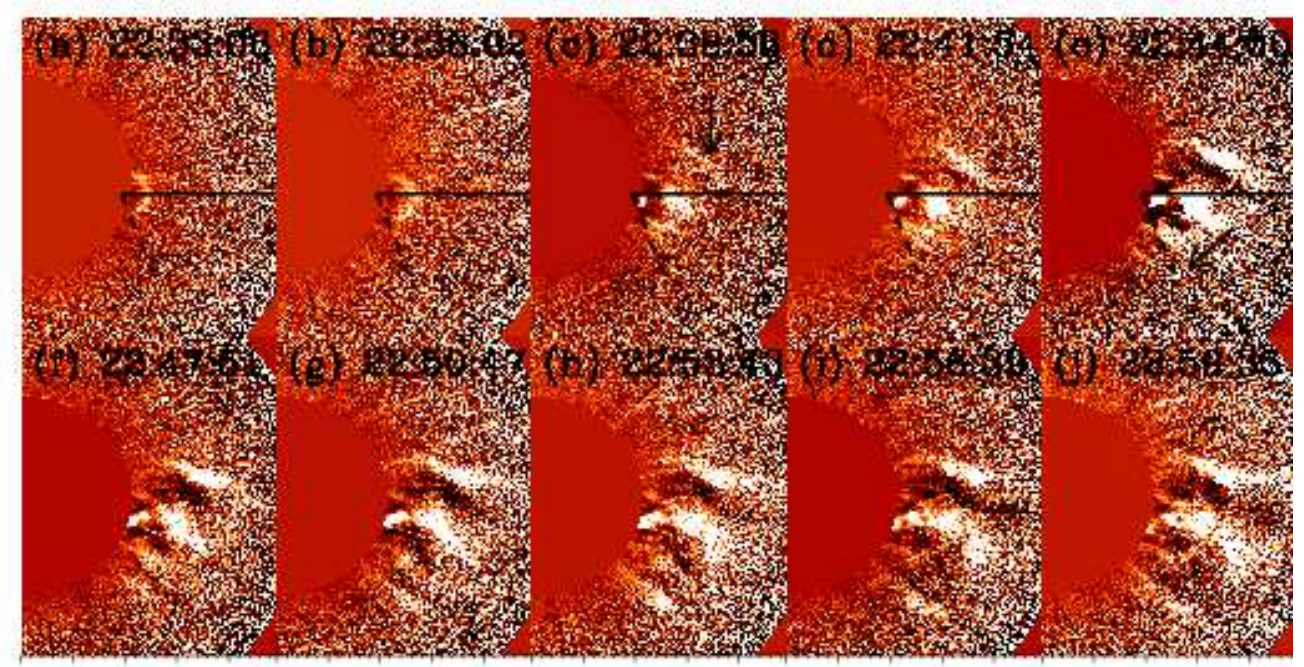

0.00 .51 .01 .52 .00 .00 .51 .01 .52 .00 .00 .51 .01 .52 .00 .00 .51 .01 .52 .00 .00 .51 .01 .52 .0 (solar radii) (solar radii) (solar radii) (solar radii) (solar radii)

Fig. 3.- Ten MK4 images for the 2003 November 1 event from 22:33:06 UT to 22:59:35 UT with 3 minutes apart. All images have subtracted the pre-eruption corona image at 22:30:06 UT. The solid and dashed lines in the upper panels are drawn to measure the heights of the expanding CME fronts.
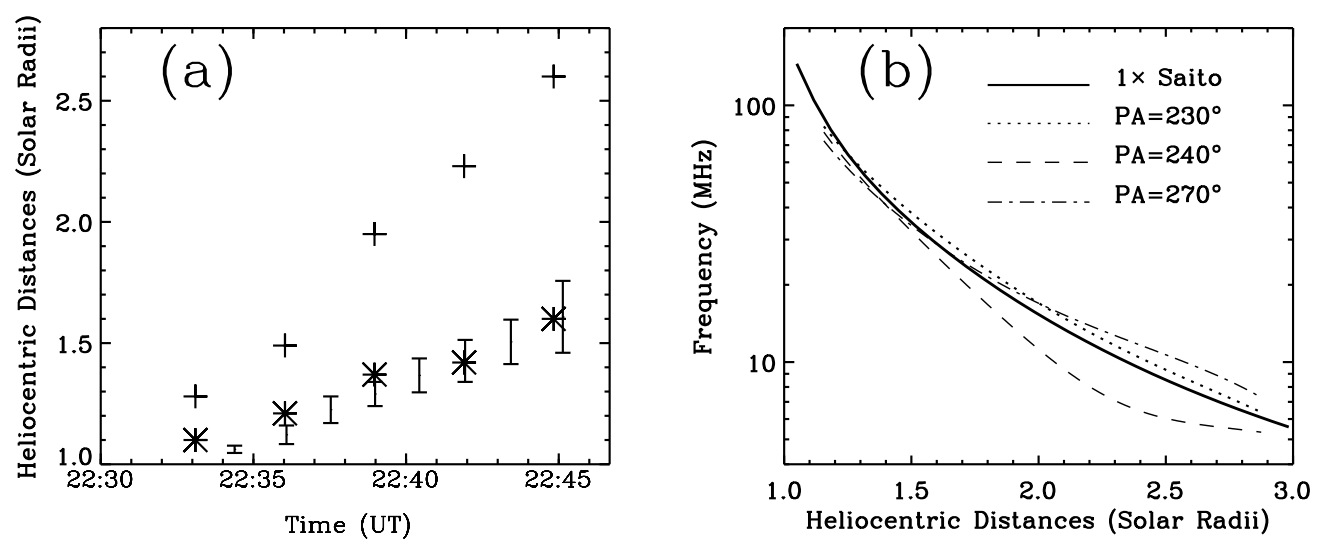

Fig. 4.- (a) Heliocentric distances of the CME front measured along the solid (pluses) and dashed (asterisks) lines plotted in the upper panels of Figure 3. The bars are given by the shock distances determined using the one-fold Saito density model (see Figure 2). (b) The radial electron density profiles deduced from the pre-eruption MK4 polarized brightness (pB) data at 22:30:06 UT, along 3 PAs at 230, 240, and 270 degrees. The one-fold Saito density model is also shown as the solid line. 


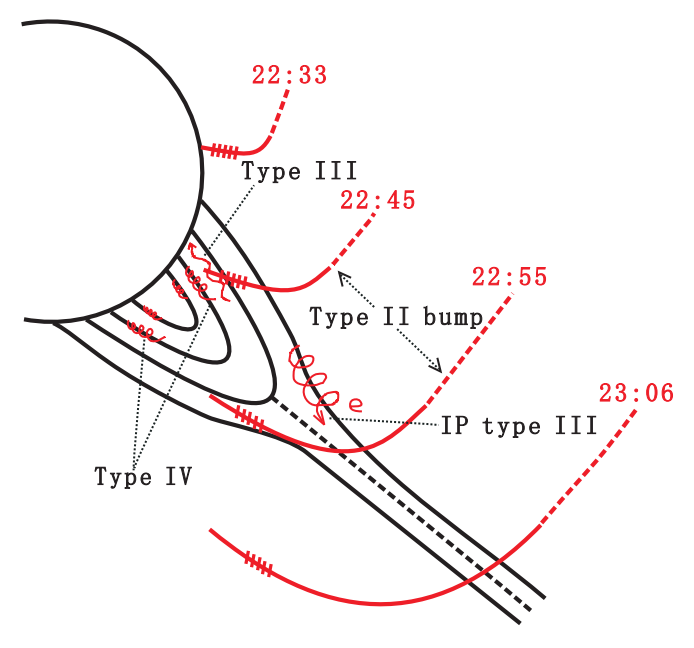

Fig. 5.- A cartoon describing the magnetic topology of the streamer structure, the outward propagation of the coronal shock wave, and the location where the type II spectral bump took place. The dashed line above the streamer cusp denotes the heliospheric current sheet. The estimated type II source regions are shown as the thick segments. The energetic electrons confined inside the closed streamer arcades, and those flowing sunwards and anti-sunwards are indicated. These electrons are related to type IV, possible RS-III, and low-frequency type III bursts. 